\title{
APPENDICE V
}

\section{SUR TAENIA TENUICOLLIS RUDOLPHI 1819 ET SON CYSTIQUE}

\author{
Par Robert-Ph. DOLLFUS
}

Lorsque un helminthologiste est en présence d'un Taenia dont les crochets ont une longueur se situant dans les environs de $20 \mu$ et qui est parasite, à l'état adulte, chez un Mustélidé, il l'identifie, le plus souvent, à Taenia tenuicollis Rudolphi 1819, nom sous lequel Rudolphi (1819, p. 159, 517) a réuni « Taenia Putorii 》 Rudolphi (1810, p. 196-197) et «Taenia Mustelae vulgaris » Rudolphi $(1810$, p. 197), espèces que Gmelin avait déjà réunies sous le nom de Taenia mustelae Gmelin 1790, p. 3068. En 1810 Rudolphi, comme en 1790 Gmelin, s'était référé uniquement aux anciennes descriptions de Goeze (1782) ; mais, en 1819, lorsqu'il proposa tenuicollis, Rudolphi s'est appuyé sur son observation personnelle de spécimens récoltés chez Mustela putorius L. 1758 et Mustela nivalis L. 1766, qu'il avait reçus de Bremser, de Vienne. Comme un peu d'incertitude subsiste au sujet de l'identité spécifique des spécimens décrits par Goeze, c'est tenuicollis, fondé sur les spécimens étudiés par Rudolphi, qui est généralement adopté, bien qu'il n'ait pas la priorité sur $T$. mustelae Gmelin.

Pour la synonymie ancienne et l'historique, je renvoie aux exposés très complets de M. S. Skinker (1935, p. 179-183) qui a adopté tenuicollis et de R. S. Freeman (1956, p. 220-226) qui a adopté mustelae.

$$
* *
$$

Si l'on compare les descriptions de tenuicollis-mustelae d'après des spécimens d'Europe à celles d'après des spécimens d'Amérique du Nord, on remarque des différences non négligeables et constantes, c'est pourquoi R. A. Wardle et J. A. Mc Leod (1952, p. 423) ont distingué « A. European tenuicollis (Thienemann 1906, etc...) 》 et « B. North American tenuicollis (Skinker 1935) 》 et précisé leurs caractères distinctifs.

Wardle et Mc Leod retiennent, pour le tenuicollis d'Europe, comme hôtes. définitifs: Mustela nivalis L. 1766, Mustela erminea L. 1766; comme hôtes 
intermédiaires : Cricetus cricetus (L. 1758), Talpa europaea L. 1758, Microtus agrestis (L. 1761), Microtus arvalis (Pallas 1778), Apodemus sylvaticus (L. 1758), Clethrionomys glareolus (Schreber 1792), Clethrionomys rufocanus (Sundevall 1846) (1).

Pour le tenuicollis d'Amérique du Nord, comme hôte définitif: Mustela vison Schreber 1777; comme hôtes intermédiaires: Ondatra zibethica (L. 1766), Ondatra spatulata (Osgood 1900).

Depuis lors, l'adulte a été signalé par R. Rausch (1952, p. 432) chez Mustela erminea arctica (Merriam) en Alaska, avec comme hôtes intermédiaires: Clethrionomys rutilus dawsoni (Merriam), Lemmus trimucronatus alascensis (Merriam), Microtus miurus paneaki Rausch.

G. A. Schad (1954, p. 221, 223, fig. 6-7), dans le Nord du Canada, a trouvé la larve ( 42 crochets de 16-18 $\mu$ ) dans le foie de Clethrionomys gapperi (Virgors 1830) ainsi que (ibid., p. 223) chez Peromyscus maniculatus (Wagner 1845), Microtus pennsylvanicus (Ord 1815) et Synaptomys borealis (Richardson 1828).

Betty Locker (1955, p. 53-54) a trouvé l'adulte chez Mustela erminea invicta Hall 1915 et $M$. vison Schreber 1777 du Montana; elle a récolté des cystiques chez Ondatra zibethica (L. 1766) et Microtus oeconomus (Pallas 1776) de l'Alaska, chez Ondatra zibethica osoyoosensis (Lord 1863), Microtus pennsylvanicus modestus (Baird 1858) et Peromyscus maniculatus artemisiae (Rhoads 1894) du Montana, chez Aplodontia rufa (Rafinesque 1817) et Peromyscus maniculatus rubidus Osgood 1901 de l'Orégon.

L'adulte a été trouvé par R.S. Freeman (1956, p. 226) chez Mustela erminea L. du Minnesota et de l'Algonquin Park et obtenu expérimentalement adulte et immature chez Mustela vison Schreber 1777, immature chez Mustela putorius L., à partir de souris, elles-mêmes infestées expérimentalement. En outre, à partir d'une souris naturellement infestée, Freeman a obtenu l'adulte chez un Mustela vison Schreber. La larve a été identifiée par Freeman (1956, p. 229-230) chez dix espèces de Rongeurs de l'Ontario et du Minnesota: Citellus franklini (Sabine 1822), Eutamias minimus (Bachmann 1839), Marmota monax (L. 1758), Tamias striatus (L. 1758), Clethrionomys gapperi (Virgors 1830), Microtus pennsylvanicus (Ord 1815), Peromyscus spp. [leucopus (Rafinesque 1818) et maniculatus (Wagner 1845)], synoptomys cooperi Baird 1858, Zapus hudsonius (Zimmermann 1780). La localisation principale des kystes était le foie.

G. Lubinsky (1957, p. 624), en Alberta, a trouvé le cystique chez Microtus pennsylvanicus (Ord 1815), Pedomys ochrogaster (Wagner 1842), Clethio-

(1) Plusieurs autres hôtes sont connus, en Europe et Asie occidentale, pour la larve et pour l'adulte. Ia. D. Kirschenblatt $(1940$, p. 555), relate avoir identifié le cystique chez Microtus socialis satunini (Ognev 1924) dans la région de Tiflis (Géorgie) et chez Chionomys nivalis (Martins 1842) à Nalbandié (Arménie russe). 
nomys gapperi (Virgors 1830), Thomomys talpoides (Richardson 1828). A. Rausch (1957, p. 365) a identifié la larve chez Synaptomys en Alaska. D'autres récoltes d'adultes et de cystiques ont été signalées par d'autres auteurs aux Etats-Unis et au Canada, mais, comme elles ne sont pas accompagnées de nouveaux renseignements, je ne m'y attarderai pas.

Un point sur lequel il y a lieu d'insister, c'est celui de la monocéphalie des cystiques d'Europe et celui de la polycéphalie habituelle des cystiques d'Amérique du Nord. Cela, plus encore que les différences dans les dimensions des crochets, justifie une forme, ou même une sous-espèce * tenuicollis americana 》 ou « mustelae americana».

Les auteurs nord-américains ont remarqué que les cystiques qu'ils trouvaient étaient des cœnures ou en majorité des cœnures.

M. S. Skinker (1935, p. 184), dans chacun des foies d'Ondatra $z$. qu'elle a examinés, a trouvé, en même temps, des cystiques à un seul scolex et des cystiques à plusieurs scolex (2) (42-60 crochets de 16-20 $\mu$ pour le cercle externe et 16-18 $\mu$ pour le cercle interne). D. J. Ameel (1942, p. 267-270, fig. 1-7), chez des zibethica du Michigan, n'a trouvé que des cystiques polycéphales; il ne les a pas rapportés à une espèce déterminée (44-46 crochets mesurant, dans un cas, 14,49 à $17,25 \mu$ pour le cercle externe et 12,4 à 15,2 pour le cercle interne; dans un second cas, 14,49 à 17,9 pour le cercle externe et 13,1 à $15,18 \mu$ pour le cercle interne) (3). Betty Locker (1955, p. 54, fig. 1-2, foies d'O. $z$. avec cœnures) a trouvé tantôt des monocéphales, tantôt des polycéphales, tantôt les deux formes (44-48 crochets, grands $15,5-18 \mu$, petits $14,0-16,5)$. R. S. Freeman (1956, p. 231-233, fig. 6, 8-9) a trouvé de 1 à 21 scolex par vésicule, tantôt seulement des monocéphales, tantôt seulement des polycéphales, tantôt à la fois des monocéphales et des polycéphales.

Parmi les cystiques de tenuicollis trouvés en Europe (désignés sous le nom Cysticercus talpae Rud. ou autrement), aucun n'a été décrit comme polycéphale.

M. S. Skinker (1935, p. 184) relate, à tort, que Villot (1883) a observé que l'on peut trouver plusieurs scolex dans une vésicule de Cysticercus talpae Rud. Le texte de Villot a été mal compris par Skinker et Villot n'a jamais dit qu'il avait observé lui-même $C$. talpae Rud. Tout au contraire, Villot (1883, p. 21) a mentionné $C$. talpae Rud. parmi les espèces dont la vésicule

(2) Pour les cystiques, Skinker a adopté le binôme Cysticercus talpae Rudolphi 1819 , nom proposé par Rudolphi $(1819$, p. 181) pour un cystique qu'il n'a pas examiné, mentionné dans le catalogue du Musée de Vienne, trouvé dans le foie de Talpa europaea L. Rien ne justifie l'attribution de Cysticercus talpae Rud. à Taenia tenuicollis Rud., Küchenmeister l'a annoncée dans un Congrès, en septembre 1856 , à Vienne (voir Küchenmeister 1858 , p. 254), sans apporter le moindre argument.

(3) D. J. Ameel (1942, p. 270) a rapproché ses cystiques de ceux, non identifiés, trouvés par Benjamin Schwartz $(1928$, p. 67) (crochets de $15 u$ ) dans le foie et autres organes de Sciurus carolinensis Gmel. du Maryland et de Virginie. Chaque cystique avait une ou plusieurs têtes. 
peut présenter des diverticules sans que ceux-ci soient un indice de prolifération et forment des scolex; ces diverticules, dit-il, peuvent n'être que des déformations purement mécaniques (c'est-à-dire des artéfacts) et la vésicule monocéphale ne devient pas un cœnure.

Parmi les nombreux observateurs du cystique de tenuicollis en Europe, il n’y en a pas, à ma connaissance, qui en aient signalé comme étant du « proliferating type »; c'est une erreur de Skinker.

Les cystiques que Max Braun (1905, p. 54) obtint expérimentalement -dans le foie de souris blanches de laboratoire (à qui il avait fait ingérer des proglottis gravides d'un tenuicollis trouvé chez un Mustela putorius L. de Prusse orientale) n'étaient pas des cœnures; si Braun avait obtenu des cœnures, il l'aurait dit (4). J. Thienemann (1906, p. 244) a étudié à nouveau les cystiques expérimentaux de Braun; il ne s'agissait pas de cœnures.

Kurt Wolffhügel (1907, p. 207-210) a décrit en détail deux cysticerques .de tenuicollis du foie de Cricetus frumentarius Pallas $1811=$ Cricetus cricetus (L. 1758), capturés en Saxe ( 72 crochets longs de 19,2 à $24 \mu$, avec des différences de forme entre les crochets d'une couronne et ceux de l'autre) ; ces cysticerques n'étaient pas des cœnures.

Tom Warwick $(1936$, p. 396, 397, 400), qui a examiné de nombreux cystiques de tenuicollis du foie d'Ondata zibethica (L.) des Shropshire, Perthshire et Stirlingshire (dans un cas, plus de cent cystiques dans le même foie) et quelques-uns du foie d'Arvicola amphibius (L.) du Shropshire, n'a pas dit avoir trouvé de cœnures.

J'ai personnellement examiné plus de 40 cystiques de tenuicollis du foie de Clethrionomys et de Pitymys de la région de Nancy (Meurthe-et-Moselle) et de Clethrionomys de Richelieu (Indre-et-Loire) ; tous ces cystiques étaient monocéphales.

En Europe, le cystique de Taenia tenuicollis Rud. a été découvert, dit Rudolph Leuckart (1856, p. 32, note 2, et p. 69, 70), par Küchenmeister, dans les canaux biliaires du Campagnol. Ce même cystique a aussi été trouvé chez le Campagnol (Hypudaeus $=$ Arvicola) par Leuckart qui le nomma * Cysticercus innominatus Hypudaei » R. Leuckart (1857, p. 208). Ayant trouvé des cystiques dans le foie de Taupes, Küchenmeister (1858, p. 254) les rapporta à Cysticercus talpae Rud., sans en donner de description, disant seulement qu'il s'agissait de la même espèce que chez le Campagnol: Cysticercus innominatus Hypudaei Leuckart, cystique que lui-même et Leuckart avaient en même temps reconnu correspondre à Taenia tenuicollis Rud. des Putois et Belettes.

(4) L'expérience de Braun a été très brièvement commentée par Skinker (1935, p. 184-185), mais avec une référence inexacte : Skinker renvoie par erreur à une publication de Braun en 1896, qui ne concerne pas tenuicollis. En 1896, dans deux notes, Max Braun $(1896$, p. $580-586 ; 1896$, p. $417-420)$ rapporte qu'à partir d'un cystique prolifère trouvé en abondance chez un Spermophilus citellus ( $L$. 1766) du jardin zoologique de Königsberg, il a obtenu expérimentalement Taenia crassiceps (Zeder 1800) chez trois jeunes Vulpes vulpes (L.). 
Depuis lors, beaucoup d'auteurs ont cité Cysticercus talpae Rud. commeétant la larve de tenuicollis (par exemple R. Leuckart 1863, p. 224 ; A. Villot. 1883 , p. 23 ; M. Braun 1898 , p. 1559 ; etc...). B. Galli-Valério (1931, p. 99). a préféré, à bon droit, l'appellation Cysticercus hypudaei Leuck. pour cecystique, qu'il a identifié chez Arvicola (Microtus) arvalis (Pallas), à Glion (Suisse).

Aucune description n'ayant été publiée des cystiques du Musée de Viennequi ont été nommés Cysticercus talpae Rud., rien ne prouve, remarque: R. Moniez (1880, p. 76), que « la forme observée chez le Campagnol est. bien la même que celle de la Taupe ». Cela n'a jamais été vérifié.

Tous les cystiques trouvés chez Talpa europaea L. ne sont pas obligatoirement Cysticercus talpae Rud. En particulier le cystique prolifère de Hausmann, étudié par H. Bendz (1842, 1843), réétudié par H. Krabbe (1862), n'est pas le même cystique que celui désigné par Küchenmeister commeétant C. talpae Rud., ainsi que l'a rappelé Max Braun (1896, p. 586 et 1896, p. 419). En outre, Küchenmeister n'a pas apporté la preuve que le cystique qu'il a désigné sous ce nom était le même que celui nommé par Rudolphi. Nous rejetons donc l'appellation Cysticercus talpae Rud. pour le cystiquede $T$. tenuicollis Rud.

Forme, dimensions et nombre des crochets. - Pour le tenuicollis d'Europe, l'iconographie des crochets est très réduite. Les références de base sont :

1845. « Ténia de la Belette. Taenia tenuicollis Rud. »: F. Dujardin, p. 558 ; Atlas, p. 14, pl. XII, fig. B 1-B 2, partie d'une double couronne et crochet isolé. Chez Mustela nivalis L. à Rennes.

1856. « Taenia tenuicollis Rud. » : Rud. Leuckart, p. 69-70, 161, pl. II, fig. $1 t$, crochet d'un scolex d'un cystique envoyé par Küchenmeister.

1884. « Taenia tenuicollis Rud. » : O. von Linstow, p. 143-144, pl. X, fig. $35 a, 35 b$, grand et petit crochet d'un adulte chez Mustela putorius L.

1906. « Taenia tenuicollis Rud. »: Joh. Thienemann, p. 245-246, pl. XV, fig. 4-5, grand et petit crochet d'un des cystiques obtenus expérimentalement par Braun chez des souris blanches.

1934. * Taenia tenuicollis Rud. 1809 (5), var. nova armata : Ch. Joyeux et J. G. Baer, p. 163 , fig. 3 , un des 52 crochets de $20 \mu$ de la var. armata, de Mustela erminea L., d'Allemagne.

Les figures de crochets de tenuicollis données par F. Dujardin, R. Leuckart, O. v. Linstow, J. Thienemann ont été reproduites juxtaposées par M. S. Skinker (1935, p. 181, fig. II, $7 a-11$ ). Sur la même figure II, 1-6, Skinker a représenté 6 crochets de cystiques de tenuicollis (americana) pour montrer la varia-

(5) 1809 est une erreur typographique pour 1819. 
tion. La forme que Skinker considère comme typique est représentée p. 178, fig. I, 4. L'hôte est Ondatra zibethica (L.), d'une ferme des environs de Kirkfield (Ontario). Il y avait parfois un, parfois deux ou trois, parfois beaucoup de scolex dans une seule vésicule.

Pour l'iconographie de crochets de spécimens nord-américains, on pourra aussi se reporter à : D. J. Ameel [1942, p. 269, fig. $4 a-d$ et $7 a-d$, grands et petits crochets de cystiques polycéphales d'O. zibethica (L.) du Michigan]; R. S. Freemann [1956, p. 233, fig. 2 A-C, groupes de crochets de trois scolex d'une même larve obtenue expérimentalement chez une souris blanche ; fig. 3, séries de crochets consécutifs d'un scolex d'une larve polycéphale d'un Clethrionomys gapperi (Virgors 1830) infesté naturellement, du Minnesota ou de 1'Ontario].

Les diverses descriptions et figures de crochets sont loin d'être concordantes et il n'y a pas non plus unanimité au sujet de leur nombre et de leurs dimensions.

Rud. Leuckart (1856, p. 70) a observé les crochets d'un scolex (préparation reçue de Küchenmeister) et en a figuré un ; il n'a pas constaté de différence de longueur et de forme entre les crochets des deux rangées; leur longueur est de 0,02 ; la distance de l'extrémité de la garde à celle de la lame est de 0,011 , à celle du manche, 0,017 ; la garde est presque aussi longue que la lame et que le manche; la lame est fortement courbée, de même que le manche, qui a, dans toute sa longueur, à peu près la même largeur; dorsalement, la limite entre la lame et le manche est marquée par une encoche très manifeste, de sorte que le dos du crochet présente deux convexités consécutives d'à peu près égale longueur. Entre la garde et le manche, il y a un large écartement, de sorte que la courbe entre eux est un arc de cercle.

F. Dujardin $(1845$, p. 558$)$ a compté \& 52 crochets caducs, longs de $0,02 \mathrm{~mm}$., très recourbés et formant une double rangée ; ses figures B 1 et B 2 sont malheureusement très petites. D'après Küchenmeister (1856, p. 320), la forme des crochets n'a, « assurément », pas été exactement représentée par Dujardin.

Thienemann, qui a compté sur ses préparations toujours 50 crochets, a constaté de petites différences de forme et de dimensions entre ceux de la rangée externe et ceux de la rangée interne. Pour les grands crochets, il a mesuré une longueur totale de $16,38 \mu$, pour les petits $13,86 \mu$, mais ces crochets n'avaient presque certainement pas atteint leurs dimensions définitives chez les souris de laboratoire, infestées expérimentalement. Pour Thienemann, ce qui caractérise les crochets de tenuicollis, c'est le sinus séparant le dos de la lame du dos du manche. Ce sinus, dit-il, est beaucoup plus prononcé chez les petits crochets que chez les grands. Le grand crochet qu'il figure (fig. 4) a une garde beaucoup plus grosse et robuste que le petit crochet de sa figure 5. Ces crochets ont un habitus très différent de ceux de Dujardin, Leuckart et Linstow ; ils n'avaient probablement pas la forme normale, c'est-à-dire la plus habituelle, celle que l'on observe dans les infestations naturelles. 
Ch. Joyeux et J. G. Baer (1934, p. 163) ont compté 52 crochets de $20 \mu$ et. disent: « la forme est bien celle représentée par Dujardin, avec l'encoche caractéristique, sur le bord opposé à la garde », mais le crochet qu'ils figurent. n'a pas la même forme que celui de la figure B 2 de Dujardin; leur figure 3 . montre un léger sinus au même niveau que le bord postérieur de la garde, alors que la figure de Dujardin montre une encoche au niveau du bord antérieur de la garde, celle-ci étant plus grosse et plus courte ; en outre, le manche est figuré, par Dujardin, un peu courbé en direction dorsale à son extrémité postérieure, ce qui n'a pas lieu chez le crochet de Joyeux et Baer, et ce: crochet diffère sensiblement de celui figuré par Leuckart comme de ceux figurés par Thienemann, mais il se rapproche beaucoup de ceux figurés par Linstow.

La variation dans la forme des crochets est aussi très étendue chez tenuicollis americana, comme le montrent les figures 1-6 de la page 181 de Skinker:

J'ai personnellement examiné, mesuré et compté les crochets de plusieurs: cystiques et adultes. Chez un cystique du foie de Pitymys subterraneus (Selys: Longch.) de Lorraine, j'ai compté 52 crochets d'environ $20 \mu$; je n'ai pu voir ces crochets que de face (vue apicale); l'extrémité postérieure du manche est élargie en spatule large d'environ $5,7 \mu$. Un cystique du foie d'un Clethrionomys glareolus (Schreber), de Lorraine, portait 46 crochets d'environ $20 \mu$, mais, en mesurant beaucoup de crochets, j'en ai trouvé dont la longueur totale (dela pointe de la lame à l'extrémité du manche) atteignait $23,5 \mu$, et d'autres seulement $18,5 \mu$; peut-être n'appartenaient-ils pas à la même rangée ou n'étaient-ils pas bien à plat. La distance de l'extrémité du manche à l'extrémité de la garde est toujours plus faible que la longueur totale; elle est, par exemple, de $19 \mu$ pour un crochet long de $21 \mu$, de $19,5 \mu$ pour des crochets longs. de $20 \mu$ à $23 \mu, 17 \mu$ pour un crochet de $18,5 \mu$.

Un second cystique de la même espèce d'hôte, de Lorraine, ne portait que' 36 crochets, longs, en moyenne, de $19 \mu$; la garde de quelques-uns avait son extrémité nettement bilobée.

Un troisième cystique de la même espèce d'hôte, de Lorraine, portait 64 crochets de 22 à $23 \mu$; la garde de quelques-uns était légèrement bilobée. Un quatrième (même espèce d'hôte, de Lorraine) portait 52 crochets.

A Richelieu (Indre-et-Loire), j'ai examiné quatre cystiques du foie d'un même Clethrionomys glareolus (Schreber); ils avaient tous les quatre 52 crochets, mesurant, dans une rangée, $23 \mu$, dans l'autre rangée de 20,5 à $21 \mu$.

J'ai aussi examiné, à Richelieu, des adultes ayant conservé une partie de leurs crochets (6) ; l'un d'eux avait encore 46 crochets, dont les grands étaient

(6) En prélcvant avec beaucoup de précaution les adultes dans l'intestin de l'hôte et en les déposant aussitôt sur une lame, on arrive généralement à obtenir des scolex ayant conservé une partie de leurs crochets. Quelques auteurs (J.-G Baer, 1902, p. 47 ; Ch. Joyeux et J.-G. Baer, 1934, p. 162, 164) ont autrefois supposé que les adultes étaient dépourvus de crochets, ceux-ci disparaissant au cours du développement, mais cette supposition est maintenant abandonnée.

La disparition précoce des crochets chez les cystiques obtenus expérimentalement par Max. Braun chez des souris blanches de laboratoire, était vraisemblablement la conséquence de conditions anormales dans un hôte anormal. 




FIG. 136. - Clethrionomys glareolus (Schreber), de Lorraine.

A. -18 crochets d'un cystique du foie.

B. - Groupe de 5 crochets d'un cystique d'un deuxième individu. C. -8 crochets d'un cystique d'un troisième individu. 

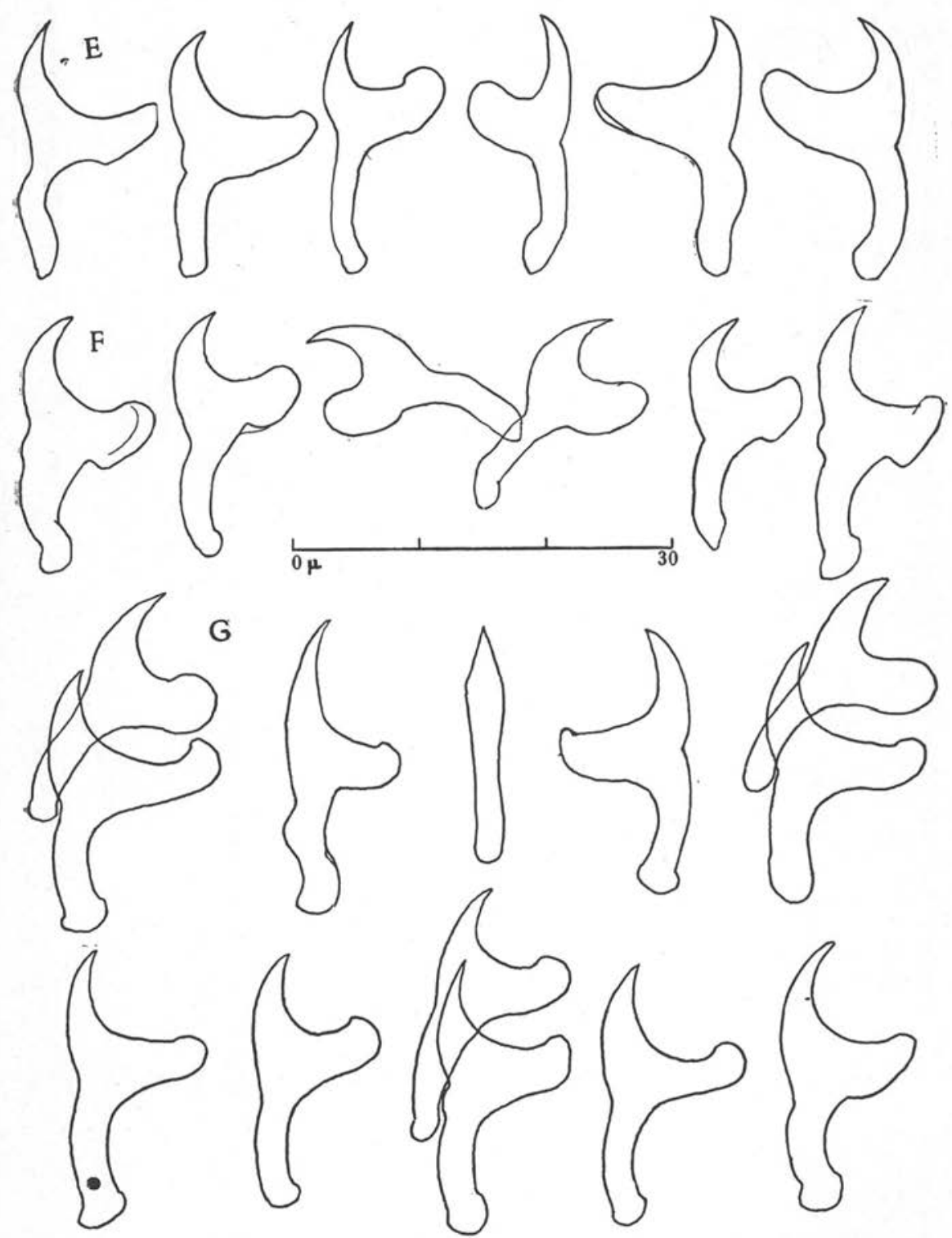

Fis. 136 E. -6 crochets d'un adulte de l'intestin d'un Mustela nivalis L. de Richelieu (7-8-1952).

Fig. 136 F. -6 crochets d'un adulte de même provenance (7-9-1957).

FIG. 136 G. - 13 crochets d'un adulte de même provenance (27-8-1959). 


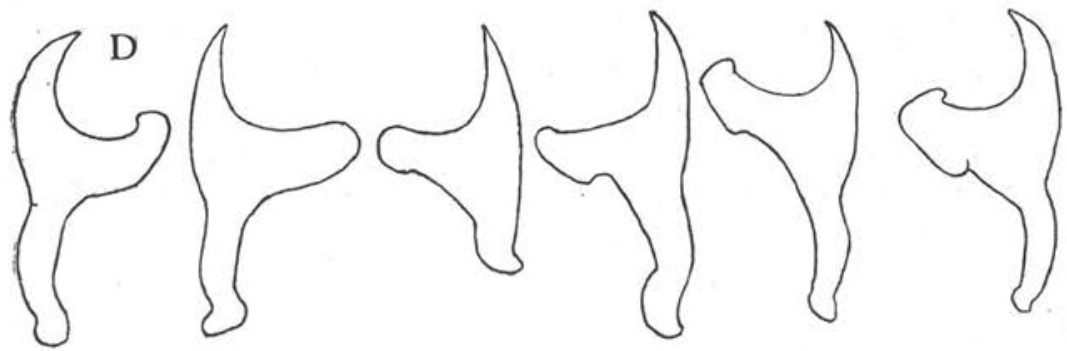

Fig. 136 D. -6 crochets d'un cystique du foie d'un Clethrionomys glareolus (Schreber), de Richelieu (30-8-1950).



Fig. 137. - Proglottis d'un adulte de même provenance (26-7-1935). 
longs de $22,6 \mu$ et les petits de $21 \mu$. Chez un autre individu, les grands atteignaient $23 \mu$ et les petits $21,3 \mu$.

Les figures que je donne d'après des cystiques et des adultes montrent combien les variations de forme sont étendues; elles affectent la lame, la garde et le manche (fig. 98-99 et $136 \mathrm{~A}-\mathrm{G}$ ). Il y a même des crochets chez lesquels le sinus du dos de la lame, considéré comme caractéristique, n'existe pas.

Je n'ai jamais compté moins de 36 crochets et plus de 64, mais Kurt Wolffhügel (1907, p. 208) en a compté 72 chez chacun des deux cystiques d'hamsters de Saxe qu'il a étudiés, et je crois que 72 est le maximum observé, du moins en Europe, de même que la longueur de $24 \mu$. Chez la sous-espèce americana, le nombre des crochets est aussi très variable; R. S. Freemann $(1956$, p. 234,239$)$ en a compté de 30 à 84 chez ses spécimens de l'Ontario.

Adulte. - L'adulte est commun chez les Mustela nivalis L. à Richelieu (Indre-et-Loire); j'en ai récolté de 1 à 10 individus dans un même intestin ; leur longueur ne dépassait pas $370 \mathrm{~mm}$., avec une largeur de 2,2 $\mathrm{mm}$. (non compris la protubérance génitale); le diamètre des ventouses variait de 80 à $165 \mu$.

L'anatomie de mes spécimens est conforme aux descriptions données par F. Dujardin (1845, p. 558 ; Atlas, p. 14, fig. B 1-B 4), Joh. Thienemann (1906, p. 229-246, pl. XV, fig. 1-6), J.-G. Baer (1932, p. 47), Ch. Joyeux et J.-G. Baer (1934, p. 162-164, fig. 3), etc... Je n'ai rien à y ajouter; je dois toutefois dire que, dans les proglottis où j'ai compté les testicules, le nombre de ceux-ci ne dépassait pas environ 60 , alors que Thienemann (1906, p. 238) en a compté environ 114 .

\section{RÉSUMÉ}

L'espèce Taenia tenuicollis Rudolphi 1819 se présente sous deux formes qui n'ont pas la même distribution géographique : en Europe, la larve est toujours monocéphale ; en Amérique du Nord, la larve est presque toujours polycéphale. Les crochets rostraux atteignent une plus grande longueur chez la forme européenne (qui est la forme typique tenuicollis tenuicollis) que chez la forme américaine : tenuicollis ssp. americana.

\section{PRINCIPAUX OUVRAgES CITÉS}

Ameri. (Donald J.), 1942. - Two larval Cestodes from the muskrat. Transact. Amer. Microscop. Soc., v. LXI, n 3, July 1942, p. 267-271, fig. 1-7.

BAER (Jean-G.), 1932. - Contribution à la Faune helminthologique de Suisse. Revue suisse de Zoologie, Genève, t. XXXIX, $\mathrm{n}^{\circ} 1$, janv. 1932, p. 1-56. fig. texte 1-3, pl. I, fig. 1-8. 
Braun (Maximilian), 1896. - Ueber einen proliferierenden Cysticercus aus dem Ziesel. Zoolog. Anzeiger, Leipzig, XIX, Bd., n 514, 5-10-1896, p. 417420.

1896. - Helminthologische Notizen. V. Ein proliferierender Cysticercus und die zugehörige Tänie. Centralbl. f. Bakt., Parasitenkunde und Infektionskr., I, Abt., XX Bd., $\mathrm{n}^{\circ}$ 16/17, 24-10-1896, p. 580-588.

1898. - Cestodes. Bronn's Klassen und Ordnungen des Thier-Reichs, IV. Bd., Würmer., 56-57-58 Lief., Leipzig, 1898, p. 1535-1614, fig., textc 86-112, pl. LIX, fig. 1-11.

1905. - Notiz zur Entwicklung der Taenia tenuicollis Rud. Centralbl. $f$. Bakt., Parasitenkunde u. Infektionkr., I. Abt., XXXIX, Bd., Heft 1, 16-61905, p. 54-55.

Dujardin (Félix), 1845. - Histoire naturelle des Helminthes ou Vers intestinaux. Nouvelles suites à Buffon, Paris, Roret, $42^{\circ}$ Livraison, 1945 , xvr +654 p., Atlas, 15 p., pl. I-XII.

Freeman (Reino S.), 1956. - Life history studies on Taenia mustelae Gmeliu, 1790 and the taxonomy of certain Taenioid Cestodes from Mustelidae. Canadian Journ. of Zoology, XXXIV, 1956, p. 219-242, fig., texte 1-3, pl. 1, fig. 1-9.

Galli-Valerio (Bruno), 1931. - Notes de Parasitologie. Centralblatt für Balter. Parasitenkunde und Infektionskr., I Abt. Origin., Bd. CXX, Heft 1-2, 23-2-1931, p. 98-106, 1 fig., texte.

Joyeux (Charles) et Baer (Jean-G.), 1934. - Sur quelques Cestodes de France. Archives du Muséum d'Histoire Naturelle, Paris, 6 s., t. XI, 1934, p. 157171, fig. 1-4.

Kirschendiatt (Ia. D.), 1940. - Stades larvaires de Vers cestodes chez des Rongeurs de Géorgie et d'Arménie. Mitteilungen der Georgischen Abteilung der Akademie der Wissenschaften der U.R.S.S., Tbilissi, Bd. I (1940), $\mathrm{n}^{\circ}$ 7, p. 551-556, fig. 1-3.

Küchenmeister (G. Friedrich H.), 1856. - Ueber die Umwandlung der Blasenwürmer in Tänien. Wiener medizinische Wochenschrift, VI Jahrg., Nr. 20, 17-5-1856, p. 319-320.

- 1858. - Ueber Leptus autumnalis (Erntemilbe). Briefliche Mittheilung an Herrn Prof. Dr. Rokitansky. Amtlicher Bericht über die 32ste Versammlung Deutscher Naturforscher und Aerzte zu Wien in Sept. 1856, Wien, 1858 , p. 254.

Leuckart (Rudolph), 1856. - Die Blasenbandwürmer und ihre Entwicklung. Zugleich ein Beitrag zur Kenntniss der Cysticercusleber, Giessen, 1856, $4^{\circ}, \mathrm{vI}+162$ p., pl. I-III.

- 1857. - Bericht über die Leistungen in der Naturgeschichte der niedern Thiere während des Jahres 1856. Archiv. für Naturgesch., XXIII Jahrg. (1857), Bd. II, p. 165-272.

1863. - Die menschlichen Parasiten und die von ihnen herrührenden Krankheiten, Leipzig und Heidelberg, I Bd., vin + 766 p., + 1 p. errata, fig. $1-268+$ portrait frontispice.

Linstrow (Otto von), 1884. - Helminthologisches. Archiv für Naturgeschichte, L. Jahrg., I. Bd., 1884 , p. 125-145, pl. VII-X, fig. 1-35.

Lоскев (Betty), 1955. - The identification of Taenia tenuicollis Rudolphi 1819, in North America. Journ. Parasitology, v. XLI, n. 1, febr. 1955, p. 51-56, fig. 1-2.

Lubinsky (George), 1957. - List of helminths from Alberta rodents. Canadiun Journ. of Zoology, v. XXXV, 1957, p. 623-627. 
Moniez (Romain), 1880. - Essai monographique sur les Cysticerques. Travanx Institut Zoologique de Lille ef de la Station maritime de Wimereux, vol. III, fasc. 1 , Paris, $4^{\circ}, 190$ p., pl. I-III.

RAusch (Robert), 1952. - Studies on the helminth fauna of Alaska. XI, Helminth Parasites of Microtine Rodents - Taxonomic considérations. Jonrnal of Parasitology, v. XXXVIII, n 5, oct. 1952, p. 415-444, pl. I, fig. 2-7, pl. II, fig. 8-14 +1 fig., texte (map).

1957. - Distribution and specificity of helminths in microtine rodents : evolutionary implications. Evolution, v. XI, n. 3, sept. 1957, p. 361-368.

SCHAD (Gerhard A.), 1954. - Helminth parasites of mice in Northeastern Quebec and the coast of Labrador. Canadian Journ. of Zoology, v. XXXII, 1954, p. 215-224, fig. 1-9.

Schwartz (Benjamin), 1928. - The occurrence of larval tapeworms in the liver, lungs, spleen, kidneys, omentum and heart of the Squirrel (Sciurus carolinensis). Helminthological Society of Washington, 108th. meeting, 17-12-1927. Journ. Parasitol., v. XV, n. 1, sept. 1928, p. 67.

SкіккеR (Mary, Scott), 1935. - A redescription of Taenia tenuicollis Rudolphi, 1819, and its larva, Cysticercus talpae Rudolphi 1819. Parasitology, Cambridge, v. XXVII, n. 2, may 1935 , p. 175-185, fig., texte I (1-3), II (1-14).

Thienemank (Johannes), 1906. - Untersuchungen über Taenia tenuicollis Rud. mit Berücksichtigung der übrigen Musteliden-Taenien. Arch. f. Naturgeschichte, Jahrg. LXXII, Bd. I, Berlin, 1906, p. 227-248, pl. XV, fig. 1-11.

Vilıot (Alfred), 1883. - Mémoire sur les cystiques des ténias. Annales des Sciences natur. Zoologie, $6^{\circ}$ s., vol. XV, art. 4, oct. 1883, p. 1-61, pl. XII, fig. 1-13.

Warde (Robert A.) et Mc Leon (James Archie), 1952. - The Zoology of Tape worms. University of Minnesota Press, xxrv +780 p., fig. 1-149 + portrait frontispice, Minneapolis, 1952.

WARwick (Tom), 1936. - The parasites of the muskrat (Ondatra zibethica L.). Parasitology, Cambridge, v. XXVIIII, $\mathrm{n}^{\circ} 3$, July 1936, p. 395-402.

WolfphüGel (Kurt), 1907. - Cysticercus der Taenia tenuicollis Rud. aus Cricetus frum€ntarius Pall. Zeitschr. für Infektions Krankheiten, parasitäre Krankh. und Hygiene der Haustiere, Bd. II, Heft 2-3, Berlin, 9-2-1907, p. 207-210. 